\title{
Expression and significance of PTEN and Claudin-3 in prostate cancer
}

\author{
XINGLONG $\mathrm{YE}^{1}$, LIJING ZHAO ${ }^{2}$ and $\mathrm{JING} \mathrm{KANG}^{2}$ \\ ${ }^{1}$ Department of Urology Surgery, The Affiliated Hospital of Beihua University; \\ ${ }^{2}$ School of Basic Medical Sciences, Jilin Medical College, Jilin, Jilin 132001, P.R. China
}

Received November 12, 2018; Accepted March 20, 2019

DOI: 10.3892/ol.2019.10212

\begin{abstract}
Expression and significance of phosphatase and tensin homolog deleted on chromosome 10 (PTEN) and Claudin-3 in the blood of patients with prostate cancer [prostate cancer (PCa)] were investigated. Retrospective analysis of 84 cases of PCa patients confirmed by pathological diagnosis were studied, as the experiment group. Moreover, the physical examination data of 84 healthy volunteers examined in the Affiliated Hospital of Beihua University were the control group. The expression levels of blood in the PTEN and Claudin-3 of both the experiment group and the control group were determined by enzyme-linked immunosorbent assay. According to the blood expression in PTEN and Claudin-3 between both the experiment group and the control group, the test value of the ROC curve in PTEN and Claudin-3 were detected by both single detection and joint detection. The expression levels of PTEN in the experiment group were significantly lower than the control group $(\mathrm{P}<0.05)$. The expression levels of Claudin-3 were higher in the experiment group than the control group $(\mathrm{P}<0.01)$. The expression levels of PTEN and Claudin-3 in the experiment group were significantly associated with the distant metastasis of cancer cells, preoperative prostate-specific antigen levels, tumor diameter and pathological stages $(\mathrm{P}<0.01)$. The expression levels of PTEN in the pathological stage of T1-T2 group was lower than that of the T3-T4 group $(\mathrm{P}<0.01)$. The expression levels of PTEN and Claudin-3 are closely related to the distant metastasis of cancer cells, preoperative prostate-specific antigen level, tumor diameter and pathological stage. Combined detection of both PTEN and Claudin-3 can improve the specificity levels of $\mathrm{PCa}$ for diagnosis and has an important diagnostic value for PCa. It can be used as a biological indicator for PCa diagnosis, disease severity analysis and efficacy evaluation.
\end{abstract}

Correspondence to: Dr Xinglong Ye, Department of Urology Surgery, The Affiliated Hospital of Beihua University, 12 Jiefang Mid Road, Jilin, Jilin 132001, P.R. China

E-mail: r6r8a3@163.com

Key words: PTEN, Claudin-3, prostate cancer, Gleason score, pathological grade, diagnostic value, clinical stage

\section{Introduction}

Prostate cancer (PCa) is a very common tumor in the male urinary system, and is a clinically complex multifactorial disease. Approximately 22,000 American men are diagnosed with PCa each year (1). In recent years, with the growth of aging population and improvement of living conditions, both the incidence and mortality rates due to $\mathrm{PCa}$ have increased (2). PCa is a malignant tumor of prostate epithelium, with slow development of tumor cells, insidious pathogenesis and no obvious symptoms in early stage (3). The cancer is often in the late stage when there are obvious symptoms such as dysuria, hematuria, impotence and urodynia. The cancer cells have undergone distant metastasis and local infiltration, and invaded organs and tissues other than prostate capsule, therefore missing the best time for treatment (4). Studies have shown that timely and effective detection of early cancer with well-timed treatment can reduce PCa mortalities, which can be conducive to the prognosis of patients with $\mathrm{PCa}$ and reduce the recurrent rate of $\mathrm{PCa}$ patients (5). Therefore, finding effective and convenient diagnostic methods and indicators can improve the early diagnosis rate of PCa patients, also it is the key to improve the cure and survival rates of PCa patients.

Claudins is a transmembrane with connexin protein and is currently found in approximately 30 family members with molecular weights between 20 and $27 \mathrm{kDa}$ (6). Changes in the expression levels of mRNA and protein are often associated with a variety of disease pathogenesis in the body. Medical scientists have confirmed that elevated levels of Claudin-4 can lead to the development and spread of cancer cells in patients with pancreatic cancer and breast cancer $(7,8)$. Claudin-1 is overexpressed in colon cancer patients (9). Claudin-3 is a member of the Claudins connexin protein and is closely associated with the transmembrane protein. It has an important effect in the transmission and transportation of cells. Some studies have suggested that the abnormal expression of Claudin-3 is closely related to the occurrence and development of tumors (10).

Phosphatase and tensin homolog deleted on chromosome 10 (PTEN) is composed of 9 exogenous factors and 8 inclusive factors, 1212 base pairs located at zone 2 and band 3 of the long arm in human chromosome 10, with a total length of $200 \mathrm{~kb}$. The functional protein encoded is a bispecific phosphate synthase, a polypeptide chain consisting of 403 amino 
acid residues (11). PTEN is the first tumor suppressor gene with phosphatase activity, which can inhibit tumors by promoting cell apoptosis (12). This gene has a high frequency of deletions and mutations in prostate cancer (13) and has received extensive attention in recent years. PTEN can mainly inhibit the growth, invasion and metastasis of cells and the pathologic adhesion of tumor cells (14). It is involved in cell differentiation, cell attachment, cell migration and apoptosis, it can maintain the body's immune system stability and has an important effect in a variety of physiological activities (15). The abnormal expression of PTEN can induce the growth of cells, which benefits the occurrence of tumors. Also induces cell invasiveness and adhesion ability, which benefits the metastasis of tumor cells (16).

Clinically, PCa examination methods mainly include prostate biopsy guided by rectal ultrasound, digital rectal examination and nuclear magnetic resonance detection (17). With the advancement of medical science, the diagnostic rate of $\mathrm{PCa}$ in the discovery of $\mathrm{PCa}$ tumor markers such as prostate specific antigen and prostate specific membrane antigen has also improved. However, some studies have found that in the autopsy of males who died normally over the age of $70,>80 \%$ of the patients with PCa were not fully detected when they were alive (18). This suggests that further investigations into the pathogenesis of $\mathrm{PCa}$ are required in order to find more accurate examination methods for tumor markers.

This study explored the blood expression levels and significance of PTEN and Claudin-3 in patients, investigated the expression and significance of PTEN and Claudin-3 in the blood of patients with $\mathrm{PCa}$ to provide a basis for clinical practice.

\section{Patients and methods}

General information. Retrospective analysis of 84 cases of PCa patients confirmed by pathological diagnosis, and the medical records were the experiment group. Moreover, the physical examination data of 84 healthy volunteers examined in the Affiliated Hospital of Beihua University (Jilin, China) were the control group. The average age in the experiment group was 67.65 years of age, according to the Gleason scoring methods, the experiment group was divided into different subgroups. There were 3 cases in the high differentiation group (Gleason 2-4 unit), 16 cases in the medium differentiation group (Gleason 5-6 unit) and 65 cases in the low differentiation group (7-10 unit). According to the TNM staging of prostate, the experiment group was divided into 26 cases of T1-T2 stage, treated as T1-T1 subgroup, and 58 cases of T3-T4 stage, treated as T3-T4 subgroup.

The study was approved by the ethics committee of Affiliated Hospital of Beihua University. All the subjects were informed and agreed to participate in the clinical study, and informed consents were obtained.

\section{Inclusion and exclusion criteria}

Inclusion criteria. Patients with PCa diagnosed by Clinical Pathology in the Affiliated Hospital of Beihua University; age $\geq 18$ years; tumor grading and clear stage. No radiotherapy, chemotherapy and other anticancer treatments were used before serum was taken. No congenital genetic disease; perfect clinical medical records for patients were available.
Exclusion criteria. Patients who had taken antibiotics within three months before sampling; patients with liver dysfunction; autoimmune system defects, and suffering from other tumors; PCa for recurrence at admission and patients with urinary system diseases.

Main reagents and instruments. Automatic washing machine (model: RT-3100; Shanghai Tiancheng Technology Co., Ltd., Shanghai, China); automatic quantitative enzyme-labeling instrument (model: Bole 680; Shanghai Dingqian Biotechnology Co., Ltd., Shanghai, China); Claudin-3 ELISA kit (item no. PRE8808; Beijing Huaxia Ocean Technology Co., Ltd., Beijing, China); PTEN ELISA kit (item no. YD2717; Shanghai Yudu Biotechnology Co., Ltd., Shanghai, China); UV-visible spectrophotometer (model: UV1700; Shanghai Jeanqi Instrument Technology Co., Ltd., Shanghai, China); and a low speed normal temperature centrifuge (model: $3-5 \mathrm{~N}$; Hunan Hengnuo Instrument Equipment Co., Ltd., Hunan, China) were used in the present study.

Collection of specimens. All the participants in the experiment were fasted for $>8 \mathrm{~h}$ the night before blood collection. The next morning, $5 \mathrm{ml}$ of elbow venous blood was taken on an empty stomach. After standing at $30^{\circ} \mathrm{C}$ for $25 \mathrm{~min}$, the serum was separated in a centrifuge of $2,300 \mathrm{x} \mathrm{g}$ at $20^{\circ} \mathrm{C}$ and centrifugation time was approximately $15 \mathrm{~min}$. After the end of the centrifugation, it was let to stand for $10 \mathrm{~min}$. After the specimens were layered, the supernatant was carefully collected and stored in a refrigerator at $-20^{\circ} \mathrm{C}$ until re-use.

Detection of PTEN and Claudin-3 expression levels. In this experiment, the expression levels of PTEN and Claudin-3 in the blood between the experiment group and the control group were determined by enzyme-linked immunosorbent assay (ELISA).

The specimen and kit were taken out of the refrigerator and melted at $30^{\circ} \mathrm{C}$. Then 20 -fold concentration of $30 \mathrm{ml}$ of washing liquid and extra $570 \mathrm{ml}$ of distilled water were added prior to dilution in the original washing solution. Subsequently, the ELISA plate was removed. Standard solution $(50 \mu \mathrm{l})$ was poured into the wells, then $10 \mu \mathrm{l}$ of sample and $60 \mu \mathrm{l}$ of sample diluent were added to the sample wells. Chromogenic reagent $(100 \mu \mathrm{l})$ was added to each well except the blank one; the ELISA plate was removed after $60 \mathrm{~min}$ in a $37^{\circ} \mathrm{C}$ water tank and $50 \mu 1$ of washing solution was added to each well. After leaving it for $1 \mathrm{~min}$, the liquid in the well was removed and the plate was washed 5 times. Enzyme standard solution $(50 \mu \mathrm{l})$ was added to each well (except for the blank control well). The chromogenic reagent was added after $15 \mathrm{~min}$ in a $37^{\circ} \mathrm{C}$ reciprocal shaking bath and avoid colored-light for $15 \mathrm{~min}$. Then the ELISA plate was taken out and termination solution was added to stop the reaction. After the reaction was terminated for $10 \mathrm{~min}$, the absorption value of each well was measured at a wavelength of $450 \mathrm{~nm}$. The R-value was calculated according to the absorption value corresponding to the concentration of the standard product, and the product is a good when the R-value has an accuracy of $99 \%$ or more. The linear regression equation was calculated using a fully automatic enzyme-labeling instrument, and the sample concentration was calculated based on the measurement of absorption values. 
Table I. The expression levels of PTEN and Claudin-3 of different baseline data in the experiment group (mean \pm SD).

\begin{tabular}{|c|c|c|c|c|c|c|c|}
\hline Type & No. of cases & PTEN & $\mathrm{t}$-test & P-value & Claudin-3 & $\mathrm{t}$-test & P-value \\
\hline Age (year) & & & 0.936 & 0.352 & & 1.809 & 0.074 \\
\hline$<65$ & 37 & $7.61 \pm 1.35$ & & & $10.88 \pm 2.06$ & & \\
\hline$\geq 65$ & 47 & $7.30 \pm 1.62$ & & & $10.02 \pm 2.24$ & & \\
\hline Smoking & & & 0.438 & 0.662 & & 0.504 & 0.616 \\
\hline Included & 69 & $7.45 \pm 1.51$ & & & $10.46 \pm 2.46$ & & \\
\hline Excluded & 15 & $7.26 \pm 1.58$ & & & $10.11 \pm 2.33$ & & \\
\hline Alcoholism & & & 0.792 & 0.430 & & 0.470 & 0.640 \\
\hline Included & 32 & $7.14 \pm 1.46$ & & & $10.54 \pm 2.40$ & & \\
\hline Excluded & 52 & $7.41 \pm 1.55$ & & & $10.28 \pm 2.50$ & & \\
\hline $\mathrm{BMI}(\mathrm{km} / \mathrm{m})$ & & & 0.516 & 0.607 & & 0.608 & 0.545 \\
\hline$<24$ & 58 & $7.38 \pm 1.58$ & & & $10.27 \pm 2.49$ & & \\
\hline$\geq 24$ & 26 & $7.19 \pm 1.51$ & & & $10.62 \pm 2.32$ & & \\
\hline Preoperative blood glucose (mmol/l) & & & 1.166 & 0.247 & & 0.464 & 0.644 \\
\hline$<4.5$ & 47 & $7.66 \pm 1.30$ & & & $10.22 \pm 2.44$ & & \\
\hline$\geq 4.5$ & 37 & $7.29 \pm 1.61$ & & & $10.47 \pm 2.47$ & & \\
\hline Preoperative CRP (mg/l) & & & 0.711 & 0.479 & & 0.364 & 0.717 \\
\hline$<100$ & 66 & $7.24 \pm 1.56$ & & & $10.27 \pm 2.49$ & & \\
\hline$\geq 100$ & 18 & $7.53 \pm 1.43$ & & & $10.51 \pm 2.43$ & & \\
\hline Preoperative $\mathrm{Hb}$ (g/l) & & & 0.750 & 0.456 & & 0.579 & 0.565 \\
\hline$<140$ & 36 & $7.43 \pm 1.53$ & & & $10.55 \pm 2.39$ & & \\
\hline$\geq 140$ & 48 & $7.18 \pm 1.50$ & & & $10.24 \pm 2.46$ & & \\
\hline Preoperagive Alb (g/l) & & & 0.782 & 0.437 & & 0.894 & 0.374 \\
\hline$<35$ & 12 & $7.59 \pm 1.37$ & & & $11.02 \pm 1.92$ & & \\
\hline$\geq 35$ & 72 & $7.22 \pm 1.54$ & & & $10.33 \pm 2.55$ & & \\
\hline
\end{tabular}

PTEN, phosphatase and tensin homolog deleted on chromosome 10.

Statistical analysis. Data analysis was performed using SPSS 17.0 (Shanghai Yuchuang Network Technology Co., Ltd., Shanghai, China) statistical software. The measurement data were expressed as mean \pm standard deviation (Means Network). The measurement data between groups were compared by t-test. ANOVA was used for the comparison of multiple groups with LSD post hoc test. The receiver operating characteristic (ROC) curve was used to evaluate the diagnostic efficacy of serum PTEN and Claudin-3 expression on PCa. When $\mathrm{P}<0.05$, the difference was statistically significant.

\section{Results}

Expression levels of PTEN and Claudin-3 in the experiment group and the control group. The experimental results showed that the expression level of PTEN in the experiment group was 7.32, which was lower than the control group (14.58), $(t=15.560, P<0.01)$. The expression level of Claudin- 3 in the experiment group was 10.36 , which was higher than the control group (expression level of 2.43), $(\mathrm{t}=26.790, \mathrm{P}<0.01)$ (Fig. 1).

The expression levels of PTEN and Claudin-3 of different baseline data in the experiment group. The results showed that the expression levels of PTEN and Claudin-3 in the experiment group were not significantly associated with age,

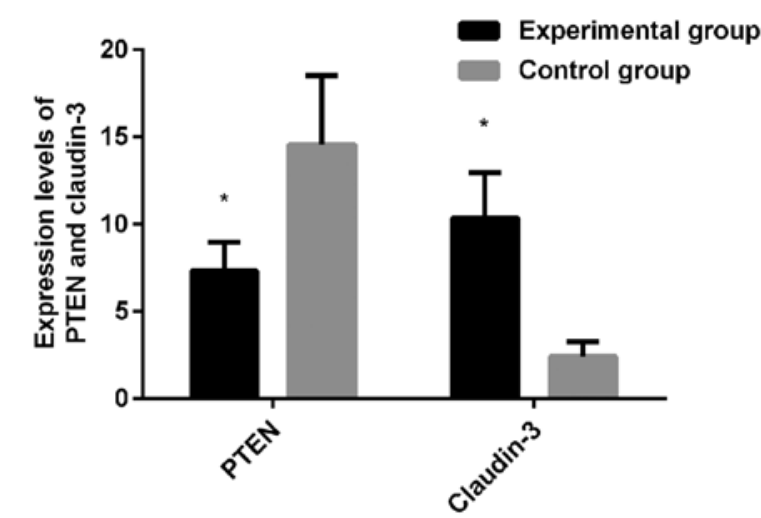

Figure 1. Comparison of PTEN expression between the experiment group and the control group. The expression levels of PTEN and Claudin-3 in the blood of the experiment group and the control group were detected by enzyme-linked immunosorbent assay (ELISA), and found that the expression level of PTEN in the experiment group was lower than the control group $(\mathrm{P}<0.01)$. The expression levels of Claudin-3 in the experiment group was higher than the control group $(\mathrm{P}<0.01)$. ${ }^{*} \mathrm{P}>0.05$, the difference is statistically significant compared with the control group. PTEN, phosphatase and tensin homolog deleted on chromosome 10 .

smoking, alcoholism, body mass index, preoperative blood glucose, preoperative $\mathrm{Hb}$, preoperative $\mathrm{Alb}$ and preoperative CRP (P>0.05) (Table I). 
Table II. Comparison of expression levels of PTEN and Claudin-3 in different clinicopathological features (mean \pm SD).

\begin{tabular}{|c|c|c|c|c|c|c|c|}
\hline Type & No. of cases & PTEN & $\mathrm{T} / \mathrm{F}$ & P-value & Claudin-3 & t-test & P-value \\
\hline Distant metastasis & & & 10.600 & $<0.01$ & & 13.380 & $<0.01$ \\
\hline Included & 41 & $6.03 \pm 0.35$ & & & $11.6 \pm 1.28$ & & \\
\hline Excluded & 43 & $7.89 \pm 1.07$ & & & $8.57 \pm 0.79$ & & \\
\hline Preoperative prostate specific antigen level & & & 2.110 & 0.04 & & 3.245 & $<0.01$ \\
\hline$<10 \mathrm{ng} / \mathrm{ml}$ & 8 & $8.03 \pm 0.93$ & & & $8.24 \pm 0.46$ & & \\
\hline$\geq 10 \mathrm{ng} / \mathrm{ml}$ & 76 & $7.01 \pm 1.33$ & & & $10.7 \pm 2.18$ & & \\
\hline Tumor diameters & & & 4.749 & $<0.01$ & & 4.715 & $<0.01$ \\
\hline$<1.5 \mathrm{~cm}$ & 31 & $8.12 \pm 0.84$ & & & $9.43 \pm 1.65$ & & \\
\hline$\geq 1.5 \mathrm{~cm}$ & 53 & $6.92 \pm 1.25$ & & & $11.2 \pm 1.71$ & & \\
\hline Pathological stages & & & 12.330 & $<0.01$ & & 29.410 & $<0.01$ \\
\hline Organ limitation & 8 & $8.01 \pm 0.95$ & & & $8.33 \pm 0.55$ & & \\
\hline Capsule invasion & 13 & $7.59 \pm 1.35$ & & & $9.12 \pm 1.24$ & & \\
\hline Seminal vesicle invasion & 21 & $6.99 \pm 1.31^{\mathrm{a}}$ & & & $11.03 \pm 1.91^{\mathrm{a}, \mathrm{b}}$ & & \\
\hline Lymph node metastasis & 42 & $6.23 \pm 0.53^{\mathrm{a}-\mathrm{c}}$ & & & $11.97 \pm 0.93^{\mathrm{a}-\mathrm{c}}$ & & \\
\hline
\end{tabular}

${ }^{a}$ The difference was statistically significant compared with the organ limitation group $(\mathrm{P}<0.05)$; ${ }^{\text {b}}$ The difference was statistically significant

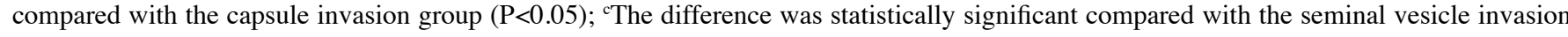
group $(\mathrm{P}<0.05)$. PTEN, phosphatase and tensin homolog deleted on chromosome 10.
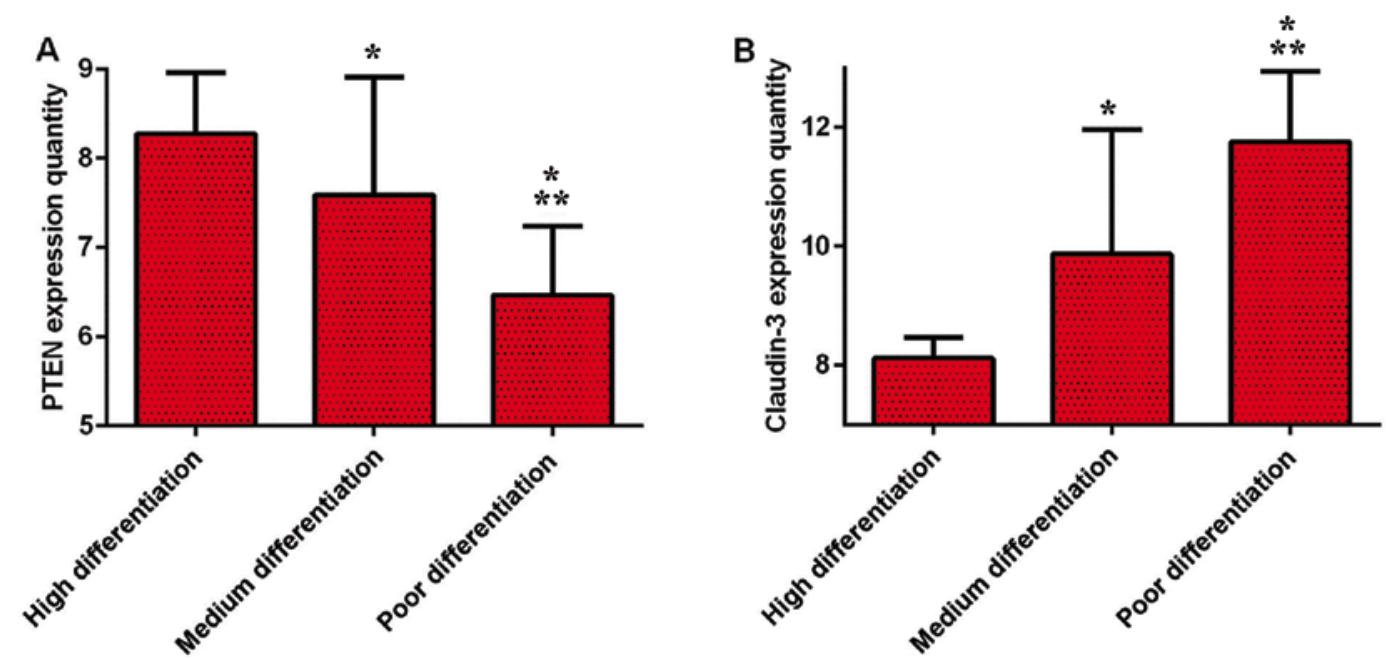

Figure 2. Comparison of the expression levels of PTEN and Claudin-3 in different clinical graded experimental groups. The expression levels of PTEN and Claudin-3 in each subgroup were detected by enzyme-linked immunosorbent assay (ELISA). (A) The expression level of PTEN in the low differentiation group was lower than that in the medium and high differentiation groups $(\mathrm{P}<0.01)$. The expression level of PTEN in the medium differentiation group was lower than that in high differentiation group $(\mathrm{P}<0.01)$. (B) The expression level of Claudin-3 in the low differentiation group was higher than that in the medium and high differentiation groups $(\mathrm{P}<0.01)$. The expression level of Claudin-3 in the medium differentiation group was higher than that in the high differentiation group $(\mathrm{P}<0.01)$. ${ }^{*} \mathrm{P}<0.05$, the difference is statistically significant compared with the high differentiation group; ${ }^{* *} \mathrm{P}<0.05$, the difference is statistically significant compared with the medium differentiation group. PTEN, phosphatase and tensin homolog deleted on chromosome 10.

Relationship between the expression levels of PTEN, Claudin-3 and the clinicopathological features. The results showed that the expression levels of PTEN and Claudin-3 in the experiment group were significantly associated with distant metastasis of cancer cells, preoperative prostate specific antigen level, tumor diameter and pathological stage $(\mathrm{P}<0.01)$ (Table II).

Expression levels of PTEN and Claudin-3 in different clinical graded experiment groups. The results showed that the lowest expression level of PTEN in the poor differentiation group was $6.46 \pm 0.78$, which was lower than the medium and high differentiation groups $(7.59 \pm 1.32$ and $8.27 \pm 0.69$, respectively) $(\mathrm{P}<0.01)$. The expression level of PTEN in the medium differentiation group was lower than that in high differentiation group $(\mathrm{P}<0.01)$. Claudin-3 had the highest expression level in the low differentiation group (11.75 \pm 1.19$)$, and was higher than both the medium and high differentiation groups $(9.87 \pm 2.09$ and $8.12 \pm 0.34$, respectively) $(\mathrm{P}<0.01)$. The expression level of Claudin-3 in the medium differentiation group was higher than that in high differentiation group $(\mathrm{P}<0.01)$ (Fig. 2). 


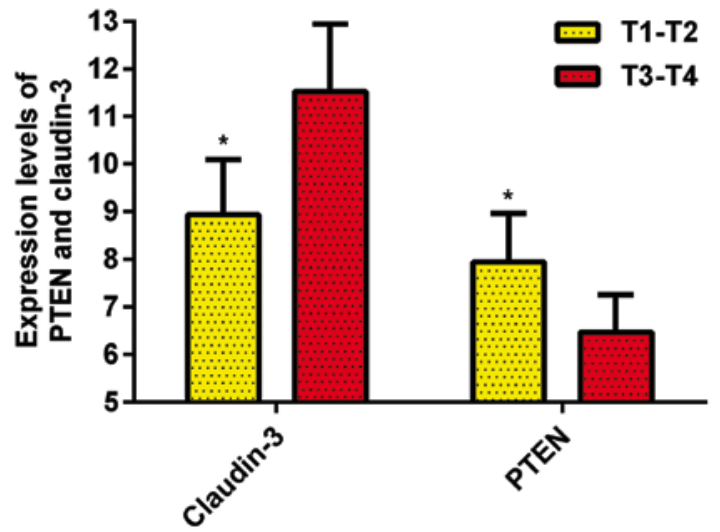

Figure 3. Comparison of the expression levels of PTEN and Claudin-3 in different TNM staging groups. The expression levels of PTEN and Claudin-3 in each subgroup were detected by enzyme-linked immunosorbent assay (ELISA). The expression level of PTEN in T1-T2 group was higher than the T3-T4 group $(\mathrm{P}<0.01)$. The expression level of Claudin-3 in the T1-T2 group was lower than the T3-T4 group $(\mathrm{P}<0.01)$. "P<0.05, statistically significant difference compared with the T3-T4 group. PTEN, phosphatase and tensin homolog deleted on chromosome 10.

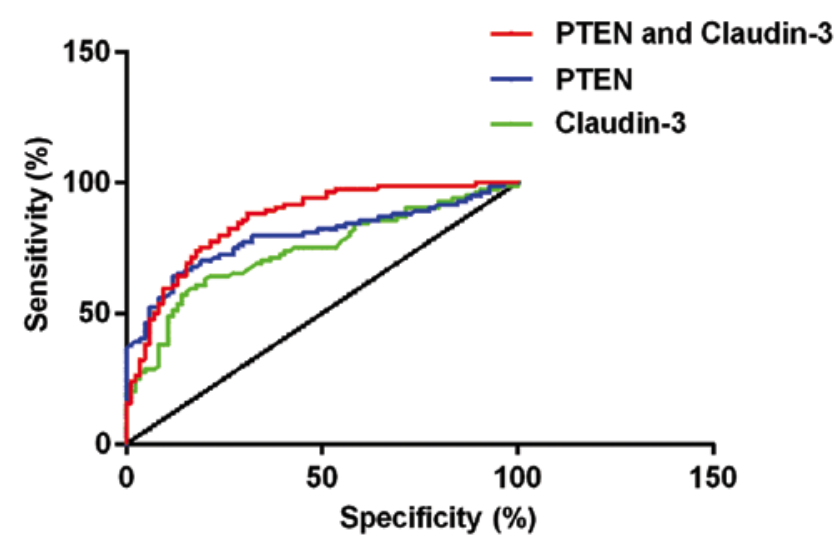

Figure 4. The AUC of PCa in PTEN was 0.7943 (95\% CI, 0.7243-0.9643), specificity was $88 \%$, sensitivity was $64 \%$ and the optimal cut-off point was 8.895. The AUC of PCa in Claudin-3 was 0.7375 (95\% CI, 0.6617-0.8133), specificity was $85 \%$, sensitivity was $57 \%$ and the optimal cut-off point was 3.310. The AUC of PCa in combined detection of PTEN and Claudin-3 was 0.8576 (95\% CI, $0.8018-0.9134)$, optimal cut-off point was 0.5697 , specificity was $69 \%$ and sensitivity was $88 \%$. PTEN, phosphatase and tensin homolog deleted on chromosome 10; $\mathrm{PCa}$, prostate cancer.

Expression levels of PTEN and Claudin-3 in different TNM stages of the experiment groups. The results showed that the expression level of PTEN in T1-T2 group was 7.94 \pm 1.02 , which was higher than the T3-T4 group $(6.474 \pm 0.79)$, there was a significant difference between the groups $(\mathrm{P}<0.01)$. The expression level of Claudin-3 in the T1-T2 group was 8.94 \pm 1.16 , which was lower than the T3-T4 group at $11.52 \pm 1.42$. There was a significant difference between the groups $(\mathrm{P}<0.01)$ (Fig. 3).

Diagnostic value of PCa in the expression levels of PTEN and Claudin-3. The expression levels of PTEN and Claudin-3 in the blood of PCa and the normal blood of healthy volunteers and the ROC curve diagram of the expression level of PTEN and Claudin-3 were evaluated. The AUC of PTEN expression level for diagnosis of PCa was 0.7943 (95\% CI, 0.7243-0.9643), the diagnostic specificity was $88 \%$, sensitivity was $64 \%$ and the optimal cut-off point for diagnosing $\mathrm{PCa}$ was 8.895 . The AUC of Claudin-3 expression level for diagnosis of PCa was 0.7375 (95\% CI, 0.6617-0.8133), the diagnostic specificity was $85 \%$, sensitivity was $57 \%$ and the optimal cut-off point for diagnosing PCa was 3.310. Further combined with PTEN and Claudin-3 to map the ROC curve diagram of $\mathrm{PCa}$, the AUC of both PTEN and Claudin-3 was 0.8576 (95\% CI, 0.8018-0.9134), and the optimal cut-off point for diagnosing PCa was 0.5697 , the specificity was $69 \%$ and sensitivity was $88 \%$ (Fig. 4).

\section{Discussion}

PCa is a unique and common tumor that occurs in males, and has the second highest incidence rate worldwide, exceeded only by lung cancer (19). The causes of PCa include inheritance, pathogenic microorganism, drugs and diet. It occurs very often in middle-aged and elderly males, and also is one of the main causes of death among them (20). The pathogenesis of $\mathrm{PCa}$ is relatively insidious, which is prone to early metastasis, and there is often no obvious specificity in the early stage. It is often at the late stage of cancer when patients have symptoms such as impotence, premature ejaculation, blood essence, dysuria and ejaculation pain. Therefore, it usually fails to achieve the desired therapeutic effect (21). PCa has a poor chemosensitivity and complicated treatment process, most of the patients are elderly, and their physical functions and organ functions have decreased to some extent. In addition, the treatment is more difficult; the side effects of late-staged cancer radiotherapy and chemotherapy are greater; older patients have poor-tolerance and poorer living conditions (22). The biological behavior of PCa is quite complicated, with the current development of PCa; the mechanism is still unclear, and finding more accurate $\mathrm{PCa}$ tumor markers is a focus for international research.

Claudin-3 is a member of the Claudins protein and is one of the most important structural molecules that make up the tight junction of epithelial cells. It mainly has an effect in maintaining intercellular barrier function and cell polarity in the body (23). Abnormal expression of Claudin-3 often leads to loss of cell polarity, and the adhesion of intercellular force is reduced to varying degrees, destroyed the epithelial permeability barrier, thereby promoting the occurrence and development of tumors. Studies have shown that in a variety of tumor patients, the expression of Claudin-3 is significantly higher than the adjacent normal tissues (10), indicating that the Claudin-3 is closely related to the occurrence and development of tumors. PTEN is a tumor suppressor gene, which relates to the growth, development and stability of cells. Irregular expression of this type of gene leads to abnormal regulation of cellular metabolism and cell cycle. Therefore, it promoted cell migration, invasion and enhanced adhesion of cells (24). There are phosphorylase activities within the PTEN, and it has the effect of inhibiting the growth and occurrence of tumors. In the occurrence and development of PCa cancer cells, it promotes apoptosis of cancer cells, inhibits the growth of cancer cells, and cancer cell adhesion and metastasis and regulates the cycles of cancer cells (25).

We have found that the expression levels of PTEN in the blood of patients with $\mathrm{PCa}$ is significantly lower than in normal people, and the difference was statistically significant 
$(\mathrm{P}<0.05)$. The results of this study are similar to the research of Yue et al (26), and they found that irregular expression of PTEN in PCa patients of human can cause abnormal accumulation of esterified cholesterol, cholesterol esterification promotes cancer cell invasion. The expression levels of Claudin-3 in the blood of patients with $\mathrm{PCa}$ was higher than in normal people, and the difference was statistically significant $(\mathrm{P}<0.01)$. The results of this study are similar to those of Chinni et al (27), who found that Claudin-3 and Claudin-4 were overexpressed in PCa patients. The expression levels of PTEN and Claudin-3 in the blood of PCa patients were significantly correlated with distant metastasis in cancer cells, preoperative prostate specific antigen levels, tumor diameter and pathological stage $(\mathrm{P}<0.01)$. Phattarataratip and Sappayatosok $(28)$ found that family members of the Claudin proteins have an important effect in oral squamous cell carcinoma. The irregular expression of Claudin-7 was associated with pathological grade, late-staged TNM grade, tumor size, fibropathopathy, vascular invasion and involvement of regional lymph nodes. Moreover, Koperek et al (29) studied the expression of PTEN protein in papillary thyroid cancer and found that the expression of PTEN is related to sex, metastasis of lymph nodes and pathological stages. This can be used as a prognostic factor for the evaluation of papillary thyroid tumors. There are some similarities between the research stated above and our studies, therefore it is confirmed that both PTEN and Claudin-3 have important effects in the growth, development and metastasis of tumors. In our further studies, we found that the lowest expression levels of PTEN in the blood of PCa patients in the low differentiation group were lower than both the medium and high differentiation groups $(\mathrm{P}<0.01)$. The highest expression levels of Claudin-3 in the low differentiation group were higher than both the medium and high differentiation groups $(\mathrm{P}<0.01)$. The expression levels of PTEN in T1-T2 group were higher than the T3-T4 group, and the difference between both groups were significant $(\mathrm{P}<0.01)$. The expression levels of Claudin-3 in T1-T2 group were lower than the T3-T4 group, and the difference between both groups were significant $(\mathrm{P}<0.01)$. By examining the ROC curve diagram of PCa between both PTEN and Claudin, we have found that the AUC of PCa was 0.8576 (95\% CI, 0.8018-0.9134); the optimal cut-off point was 0.5697 ; specificity was $69 \%$ and the sensitivity was $88 \%$; The ROC curve of PCa was diagnosed by plotting the expression levels of PTEN and Claudin-3; The combined diagnosis can improve the sensitivity levels of PCa.

This study strictly selected the research objects according to the inclusion and exclusion criteria, which ensured the reliability of the study results. However, this study still has certain defects. We only included a few patients in this study, and more PCa patients with different pathological types should be collected for research. The present study did not conduct an in-depth study of the association between PTEN, Claudin-3 and other clinical symptoms of PCa patients. Therefore, there are certain limitations.

PTEN is lowly expressed in the blood of PCa patients and Claudin-3 is highly expressed in the blood of PCa patients. The expression levels of PTEN and Claudin-3 are closely related to the distant metastasis of cancer cells, preoperative prostate specific antigen level, tumor diameter and pathological stage. Combined detection of PTEN and Claudin-3 can improve the specificity of $\mathrm{PCa}$ for diagnosis, which has an important diagnostic value for PCa. It can be used as a biological indicator for PCa diagnosis, disease severity analysis and efficacy evaluation.

\section{Acknowledgements}

Not applicable.

\section{Funding}

No funding was received.

\section{Availability of data and materials}

The datasets used and/or analyzed during the present study are available from the corresponding author on reasonable request.

\section{Authors' contributions}

$\mathrm{XY}$ was involved in writing the manuscript. XY and LZ performed ELISA. XY and JK collected the patient general data and specimens. All authors read and approved the final manuscript.

\section{Ethics approval and consent to participate}

The study was approved by the Ethics Committee of Affiliated Hospital of Beihua University (Jilin, China). Patients who participated in this research had complete clinical data. Signed informed consents were obtained from the patients or the guardians.

\section{Patient consent for publication}

Not applicable.

\section{Competing interests}

The authors declare that they have no competing interests.

\section{References}

1. Robinson D, Van Allen EM, Wu YM, Schultz N, Lonigro RJ, Mosquera JM, Montgomery B, Taplin ME, Pritchard CC, Attard G, et al: Integrative clinical genomics of advanced prostate cancer. Cell 161: 1215-1228, 2015.

2. Song W and Jeon HG: Incidence of kidney, bladder, and prostate cancers in Korea: An update. Korean J Urol 56: 422-428, 2015.

3. De Nunzio C, Albisinni S, Freedland SJ, Miano L, Cindolo L, Finazzi Agrò E, Autorino R, De Sio M, Schips L and Tubaro A: Abdominal obesity as risk factor for prostate cancer diagnosis and high grade disease: A prospective multicenter Italian cohort study. Urol Oncol 31: 997-1002, 2013.

4. Krupski TL, Stukenborg GJ, Moon K and Theodorescu D: The relationship of palliative transurethral resection of the prostate with disease progression in patients with prostate cancer. BJU Int 106: 1477-1483, 2010.

5. Liu L, Tian Z, Zhang Z and Fei B: Computer-aided detection of prostate cancer with MRI: Technology and applications. Acad Radiol 23: 1024-1046, 2016.

6. Koval M: Claudin heterogeneity and control of lung tight junctions. Annu Rev Physiol 75: 551-567, 2013. 
7. Neesse A, Hahnenkamp A, Griesmann H, Buchholz M, Hahn SA, Maghnouj A, Fendrich V, Ring J, Sipos B, Tuveson DA, et al: Claudin-4-targeted optical imaging detects pancreatic cancer and its precursor lesions. Gut 62: 1034-1043, 2013.

8. Mosley M, Knight J, Neesse A, Michl P, Iezzi M, Kersemans V and Cornelissen B: Claudin-4 SPECT imaging allows detection of aplastic lesions in a mouse model of breast cancer. $\mathrm{J}$ Nucl Med 56: 745-751, 2015.

9. Bezdekova M, Brychtova S, Sedlakova E, Langova K, Brychta T and Belej K: Analysis of Snail-1, E-cadherin and claudin-1 expression in colorectal adenomas and carcinomas. Int $\mathbf{J}$ Mol Sci 13: 1632-1643, 2012.

10. Li JY, Xie F, Xu XP, Ma JJ, Zhou DC, Liao Y, Tang J, Xie Q, Bai L and Nan QZ: Claudin-3 expression in colorectal carcinoma and its significance. Nan Fang Yi Ke Da Xue Xue Bao 37: 63-67, 2017 (In Chinese)

11. Jones N, Bonnet F, Sfar S, Lafitte M, Lafon D, Sierankowski G, Brouste V, Banneau G, Tunon de Lara C, Debled M, et al: Comprehensive analysis of PTEN status in breast carcinomas. Int J Cancer 133: 323-334, 2013.

12. Zhang Y, Feng J, Fu H, Liu C, Yu Z, Sun Y, She X, Li P, Zhao C Liu Y, et al: Coagulation factor X regulated by CASC2c recruited macrophages and induced M2 polarization in glioblastoma multiforme. Front Immunol 9: 1557, 2018.

13. Wise HM, Hermida MA and Leslie NR: Prostate cancer, PI3K, PTEN and prognosis. Clin Sci (Lond) 131: 197-210, 2017.

14. Zhang LL, Liu J, Lei S, Zhang J, Zhou W and Yu HG: PTEN inhibits the invasion and metastasis of gastric cancer via downregulation of FAK expression. Cell Signal 26: 1011-1020, 2014.

15. Huang J, Yuan SX, Wang DX, Wu QX, Wang X, Pi CJ, Zou X, Chen L, Ying LJ, Wu K, et al: The role of COX-2 in mediating the effect of PTEN on BMP9 induced osteogenic differentiation in mouse embryonic fibroblasts. Biomaterials 35: 9649-9659, 2014.

16. Yu M, Mu Y, Qi Y, Qin S, Qiu Y, Cui R and Zhong M: Odontogenic ameloblast-associated protein (ODAM) inhibits human colorectal cancer growth by promoting PTEN elevation and inactivating PI3K/AKT signaling. Biomed Pharmacother 84: 601-607, 2016.

17. Stamatiou K, Alevizos A, Karanasiou V, Mariolis A, Mihas C Papathanasiou M, Bovis K and Sofras F: Impact of additional sampling in the TRUS-guided biopsy for the diagnosis of prostate cancer. Urol Int 78: 313-317, 2007.

18. Albert RH and Clark MM: Cancer screening in the older patient. Am Fam Physician 78: 1369-1374, 2008.

19. Coakley FV, Oto A, Alexander LF, Allen BC, Davis BJ, Froemming AT, Fulgham PF, Hosseinzadeh K, Porter C, Sahni VA, et al; Expert Panel on Urologic Imaging: ACR Appropriateness criteria ${ }^{\circledR}$ prostate cancer-pretreatment detection, surveillance and staging. J Am Coll Radiol 14: S245-S257, 2017.
20. Bailey DE Jr and Wallace M: Critical review: Is watchful waiting a viable management option for older men with prostate cancer? Am J Men Health 1: 18-28, 2007.

21. de Oliveira Barros EG, Palumbo A Jr, Mello PL, de Mattos RM, da Silva JH, Pontes B, Viana NB, do Amaral RF, Lima FR, da Costa NM, et al: The reciprocal interactions between astrocytes and prostate cancer cells represent an early event associated with brain metastasis. Clin Exp Metastasis 31: 461-474, 2014.

22. Sinibaldi VJ: Docetaxel treatment in the elderly patient with hormone refractory prostate cancer. Clin Interv Aging 2: 555-560, 2007.

23. Yokoyama M, Narita T, Sakurai H, Katsumata-Kato O, Sugiya $H$ and Fujita-Yoshigaki J: Maintenance of claudin-3 expression and the barrier functions of intercellular junctions in parotid acinar cells via the inhibition of Src signaling. Arch Oral Biol 81: 141-150, 2017.

24. Yang XD, Zhao SF, Zhang Q, Li W, Wang YX, Hong XW and Hu QG: PTEN gene polymorphisms and susceptibility to oral squamous cell carcinoma in a Chinese Han population. Tumour Biol 37: 577-582, 2016.

25. Yang Y, Guo JX and Shao ZQ: miR-21 targets and inhibits tumor suppressor gene PTEN to promote prostate cancer cell proliferation and invasion: An experimental study. Asian Pac J Trop Med 10: 87-91, 2017

26. Yue S, Li J, Lee SY, Lee HJ, Shao T, Song B, Cheng L, Masterson TA, Liu X, Ratliff TL, et al: Cholesteryl ester accumulation induced by PTEN loss and PI3K/AKT activation underlies human prostate cancer aggressiveness. Cell Metab 19: 393-406, 2014.

27. Chinni SR, Li Y, Upadhyay S, Koppolu PK and Sarkar FH: Indole-3-carbinol (I3C) induced cell growth inhibition, G1 cell cycle arrest and apoptosis in prostate cancer cells. Oncogene 20: 2927-2936, 2001

28. Phattarataratip E and Sappayatosok K: Expression of claudin-5, claudin-7 and occludin in oral squamous cell carcinoma and their clinico-pathological significance. J Clin Exp Dent 8: e299-e306, 2016.

29. Koperek O, Kornauth C, Capper D, Berghoff AS, Asari R, Niederle B, von Deimling A, Birner P and Preusser M: Immunohistochemical detection of the BRAF V600E-mutated protein in papillary thyroid carcinoma. Am J Surg Pathol 36: 844-850, 2012

This work is licensed under a Creative Commons Attribution-NonCommercial-NoDerivatives 4.0 International (CC BY-NC-ND 4.0) License. 